\title{
TRIPLES ON REFLECTIVE SUBCATEGORIES OF FUNCTOR CATEGORIES
}

\author{
DAVID C. NEWELL
}

ABSTRACT. We show that if $\mathbf{S}$ is a cocontinuous triple on a full re: flective subcategory of a functor category then the category of S-algebras is again a full reflective subcategory of a functor category.

This note should be considered an addendum to [4], and definitions for all of the terminology and concepts we use can be found there.

We shall also fix $\mathbf{V}$ to be a closed bicomplete category and, in this note, all of the category theory is done relative to $\mathbf{V}$.

In [4], we have shown the following:

(I) If $\mathbf{C}$ is a small category and $\mathbf{T}$ is a cocontinuous triple on the functor category $\mathbf{V}^{C}$, then there is a small category $\mathrm{C}^{\prime}$ and a functor $f$ : $\mathrm{C} \rightarrow \mathrm{C}^{\prime}$ so that

(a) $\mathbf{T}$ is the triple induced by the adjoint pair $\left(f^{*}, f^{l}\right): \mathrm{V}^{\mathrm{C}^{\prime}} \rightarrow \mathrm{V}^{\mathrm{C}}$, where $f^{*}: \mathbf{V}^{\prime} \rightarrow \mathbf{V}^{\mathbf{C}}$ is the functor induced by $f$ and $f^{l}$ is the left adjoint of $f^{*}$;

(b) the adjoint pair $\left(f^{*}, f^{l}\right)$ is tripleable, so that there is an equivalence of categories $V^{C^{\prime}} \cong\left(V^{C}\right)^{T}$, where $\left(V^{C}\right)^{\mathbf{T}}$ is the category of $\mathbf{T}$. algebras.

(II) If $\mathbf{C}$ is a small category and $\mathbf{T}$ is any triple on $\mathbf{V}^{\mathrm{C}}$, there is a unique cocontinuous triple $\hat{\mathbf{T}}$ on $\mathrm{V}^{\mathrm{C}}$ and a map of triples $\tau: \hat{\mathbf{T}} \rightarrow \mathbf{T}$ so that, if $R: \mathrm{C}^{\circ} \rightarrow \mathrm{V}^{\mathrm{C}}$ denotes the right Yoneda embedding of $\mathrm{C}^{\circ}$ into the representable functors of $\mathbf{V}^{\mathrm{C}}, \tau R$ is the identity (we shall refer to $\hat{\mathbf{T}}$ as the cocontinuous approximation to $\mathbf{T}$ ).

In this paper, we shall prove the following

1. Theorem. Suppose $\mathrm{C}$ is a small category, $\mathrm{A}$ is a full reflective subcategory of $\mathbf{V}^{\mathrm{C}}$ (i.e. the inclusion functor of $\mathbf{A}$ to $\mathrm{V}^{\mathrm{C}}$ has a left adjoint) and $\mathbf{S}$ is a cocontinuous triple on $\mathbf{A}$. Then there is a small catego-

Presented to the Society January 25, 1973; received by the editors August 16, 1972 and, in revised form, August 6, 1973.

AMS (MOS) subject classifications (1970). Primary 18C15; Secondary 18E99, $18 \mathrm{~F} 20$. 
ry $\mathbf{C}^{\prime}$ for which the category of $\mathbf{S}$-algebras $\mathbf{A}^{\mathbf{S}}$ is a full reflective subca. tegory of $\mathrm{V}^{\prime}$.

The basic idea of the proof of this theorem is as follows: as we shall see, $\mathbf{S}$ induces a triple $\mathbf{T}$ on $\mathbf{V}^{\mathbf{C}}$ in an obvious way; we use (II) to construct the cocontinuous approximation $\hat{\mathbf{T}}$ of $\mathbf{T}$, and then we apply (I) to $\hat{\mathbf{T}}$ to obtain the desired category $\mathbf{C}^{\prime}$.

The rest of this paper is devoted to showing that the above outline does indeed give a proof for the theorem.

Proof of the theorem. Let $\mathbf{A}$ and $\mathbf{B}$ be categories and suppose $(i, r)$ : $\mathbf{A} \rightarrow \mathbf{B}$ is an adjoint pair from $\mathbf{A}$ to $\mathbf{B}$ with unit $u: 1_{\mathbf{B}} \rightarrow$ ir and counit $e:$ $r i \rightarrow 1_{\mathbf{A}^{*}}$. We shall let $\mathbf{R}=(R, u, m)$ denote the triple on $\mathbf{B}$ induced by $(i, r)$ (so that $m=i e r$ ).

Suppose $\mathbf{S}=\left(S, \eta^{\prime}, \mu^{\prime}\right)$ is a triple on $\mathbf{A}$. Then $\mathbf{S}$, together with $(i, r)$, induces a triple $\mathbf{T}=(T, \dot{\eta}, \mu)$ on $\mathbf{B}$ by letting $T=i S r, \eta=\left(i \eta^{\prime} r\right) \cdot u$ and $\mu=\left(i \mu^{\prime} r\right) \cdot(i S e S r)$. Equivalently, $\mathbf{T}$ is the triple induced by the adjoint pair obtained by composing the adjoint pair $(i, r): \mathbf{A} \rightarrow \mathbf{B}$ with the adjoint pair $\left(U^{\mathbf{S}}, F^{\mathbf{S}}\right): \mathbf{A}^{\mathbf{S}} \rightarrow \mathbf{A}$, where $U^{\mathbf{S}}: \mathbf{A}^{\mathbf{S}} \rightarrow \mathbf{A}$ is the usual "underlying" functor from the category of $\mathbf{S}$-algebras to $\mathbf{A}$ and $F^{\mathbf{S}}$ is the usual "free" functor.

One obtains easily the following facts:

(1) there is a comparison functor $\tilde{i}: \mathbf{A}^{\mathbf{S}} \rightarrow \mathbf{B}^{\mathbf{T}}$;

(2) there is a map of triples $\theta: \mathbf{R} \rightarrow \mathbf{T}$ given by $\theta=i \eta^{\prime} r$.

2. Proposition. Suppose $\mathbf{A}$ is a full reflective subcategory of $\mathbf{B}$, i.e., the inclusion functor $i: \mathbf{A} \rightarrow \mathbf{B}$ has a left adjoint $r, \mathbf{S}=\left(S, \eta^{\prime}, \mu^{\prime}\right)$ is a triple on $\mathbf{A}$, and $\mathbf{T}=(T, \eta, \mu)$ is the triple on $\mathbf{B}$ induced by $\mathbf{S}$ and $(i, r)$. Then

(a) the comparison functor $\tilde{i}: \mathbf{A}^{\mathbf{S}} \rightarrow \mathbf{B}^{\mathbf{T}}$ of (1) is an equivalence of categories, and

(b) if $\mathbf{R}=(R, u, m)$ is the idempotent triple on $\mathbf{B}$ induced by the adjoint pair $(i, r)$, then $T=T R=R T$ and the map of triples $\theta: R \rightarrow T=R T$ of (2) is given by $\theta=R \eta$. Furthermore, $\mu \cdot \theta T=\mu \cdot T \theta=1_{T}$.

Proof. (a) Follows from Beck's tripleability theorem (see [7]). For (b), we have $R T=i r i S r=i S r=T$, as the counit $e: r i \rightarrow 1_{\mathrm{A}}$ is the identity. Similarly $T R=T . R \eta=i r\left(i \eta^{\prime} r \cdot u\right)=i r i \eta^{\prime} r \cdot i r u=i \eta^{\prime} r\left(\right.$ as $r i=1_{\Lambda}$ and $r u=$ $\left.1_{r}\right)=\theta . \mu \cdot \theta T=i \mu^{\prime} r \cdot i S e S r \cdot i \eta^{\prime} r i S r=i \mu^{\prime} r \cdot i \eta^{\prime} S r$ (as $e=1$ and $r i=$ $\left.1_{\mathbf{\Lambda}}\right)=i\left(\mu^{\prime} \cdot \eta^{\prime} S\right)_{r}=i 1_{S} r($ as $\mathbf{S}$ is a triple $)=1_{T} \cdot$ Similarly $\mu \cdot T \theta=1_{T} \cdot \square$

For the rest of this paper, let us make the following hypotheses. 
(i) $\mathbf{B}$ is cocomplete and there is a small category $\mathbf{C}$ and a functor $k: \mathbf{C} \rightarrow \mathbf{B}$ which is dense in $\mathbf{B}$ (see [7]);

(ii) there is an adjoint pair $(i, r): \mathbf{A} \rightarrow \mathbf{B}$ whose counit is the identity (*) (so that $\mathbf{A}$ is equivalent to a full reflective subcategory of $\mathbf{B}$ ) and $\mathbf{R}=(R, u, m)$ is the idempotent triple on $\mathbf{B}$ induced by $(i, r)$;

(iii) $\mathbf{S}=\left(S, \eta^{\prime}, \mu^{\prime}\right)$ is a cocontinuous triple on $\mathbf{A}$ and, for $\mathbf{T}=(T, \eta, \mu)$, the triple on $\mathbf{B}$ induced by $\mathbf{S}$ and $(i, r)$, there is a cocontinuous triple $\hat{\mathbf{T}}=(\hat{T}, \hat{\eta}, \hat{\mu})$ on $\mathbf{B}$ and a map of triples $\tau: \hat{\mathbf{T}} \rightarrow \mathbf{T}$ with $\tau k=1$.

We note that if $\mathbf{C}$ is a small category, $\mathbf{A}$ is a full reflective subcategory of $\mathbf{V}^{\mathbf{C}}$, and $\mathbf{S}$ is a cocontinuous triple on $\mathbf{A}$ (as in the hypotheses of 1), then $\mathbf{B}=\mathbf{V}^{\mathbf{C}}, k$ the right Yoneda embedding $R: C^{\circ} \rightarrow \mathbf{V}^{\mathrm{C}}$, and $\hat{\mathbf{T}}$ the cocontinuous approximation of $\mathbf{T}$ satisfy the above hypotheses $(*)$.

Recall that for $\mathbf{X}$ a category and for $\mathbf{R}=(R, u, m)$ and $\mathbf{T}=(T, \eta, \mu)$ two triples on $\mathbf{X}$, the composite triple of $\mathbf{R}$ and $\mathbf{T}$ is a triple $\mathbf{R T}=(R T$, $u \eta, v)$ for which $R \eta: R \rightarrow R T$ and $u T: T \rightarrow R T$ are maps of triples and for which $v \cdot(R \eta u T)=1_{R T}$.

3. Proposition. Under the hypotheses $(*), \mathbf{T}$ is a composite triple of $\mathbf{R}$ and $\hat{\mathbf{T}}$.

Proof. $r$, being an adjoint, is cocontinuous. Since $r T=r i S r=S r$ and since $r r: r \hat{T} \rightarrow r T=S r$ is a natural transformation between cocontinuous functors for which $r \tau k=1$, and since $k$ is assumed dense, it follows that $r \tau$ is an isomorphism of functors. Hence $R r: R \hat{T} \rightarrow R T=T$ is an isomorphism of functors. Let RT be $R \hat{T}$ with the triple structure induced by that of $T$ via the isomorphism $R \tau$. Since $\hat{\eta}: \tau=\eta$, one has $(R \tau) \cdot(u \hat{\eta})=u \eta=\eta$ so that $u \hat{\eta}$ is the unit of $\mathbf{R} \hat{\mathbf{T}}$.

$R \hat{\eta}: R \rightarrow R \hat{T}$ is a map of triples, since $R \tau \cdot R \hat{\eta}=R(\tau \cdot \hat{\eta})=R \eta=\theta$ is a map of triples.

We now show that $u \hat{T}: \hat{T} \rightarrow R \hat{T}$ is a map of triples. Now $(R \tau \cdot \hat{T}) k=$ $R \tau k \cdot u \hat{T} k=1 \cdot u T k=u i S r k=1$ (as $u i=1$ and $r k=1$ ). Since $\hat{T}$ is cocontinuous and $k$ is dense, $\hat{T}$ is the left Kan extension of $\hat{T} k$ along $k$ (see $[7, p .232])$. The universal property of left Kan extensions gives us that $R \tau \cdot n \hat{T}=\tau$, and since $\tau$ is a map of triples, so is $u \hat{T}$.

Finally, if $v$ is the multiplication of $\mathbf{R} \hat{\mathbf{T}}$ (induced by $\mu$ ), we have $[v \cdot$ $(R \hat{\eta} n \hat{T})]=1_{R} \hat{T}$ since, by $\$ 2, \mu \cdot \theta T=1$ so that the diagram 


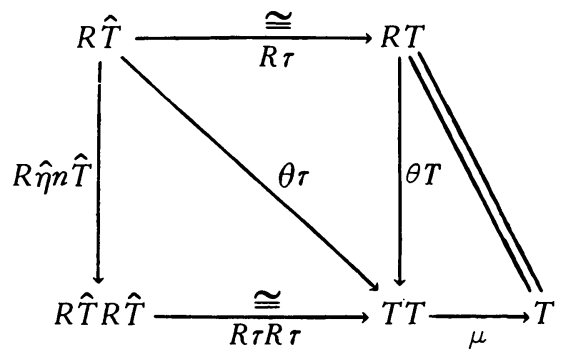

commutes.

4. Corollary. Under the hypotheses $(*), \mathbf{A}^{\mathbf{S}}$ is equivalent to a full reflective subcategory of $\mathbf{B} \hat{\mathbf{T}}$.

Proof. From [2, D. 122] and $\$ 3$ we have a lifting of $R$ to a triple $\widetilde{R}$ on $\mathbf{B}^{\hat{\mathbf{T}}}$ and an isomorphism of categories $\Phi:\left(\mathbf{B}^{\hat{\mathbf{T}}}\right)^{\hat{\mathbf{R}}} \simeq \mathbf{B}^{\mathbf{R} \hat{\mathbf{T}}}$. But $\mathbf{R} \hat{\mathbf{T}} \cong \mathbf{T}$

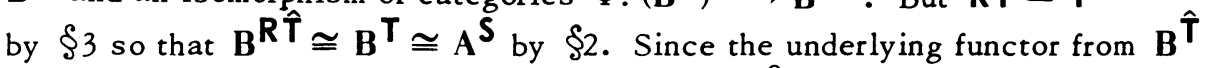
to $\mathbf{B}$ is faithful and $\mathbf{R}$ is idempotent, the lifting $\widetilde{\mathbf{R}}$ is idempotent.

We note that Theorem 1 now follows from this corollary.

A problem arising from this theorem is the following: if $\mathrm{C}$ is a small category, $A$ is a full reflective subcategory of $\mathbf{V}^{C}$, and $\mathbf{S}$ a cocontinuous triple on $A$, then is $A^{S}$ a full reflective subcategory of $V^{C}$ ' "of the same type"'? For example, if $\mathbf{V}=\mathbf{A b}$ (the category of abelian groups), $\mathbf{C}$ a small abelian category, $\mathcal{Q}$ the category of left exact functors from $\mathbf{C}$ to $\mathbf{A b}$, and $S$ a cocontinuous triple on $\mathcal{L}$, then $\mathcal{L}$ is a full reflective subcategory of $\mathbf{A b}^{C^{\prime}}$ for some preadditive category $\mathbf{C}^{\prime}$ by our theorem, but is $\mathbf{C}^{\prime}$ abelian and is $\mathcal{L}^{\mathbf{S}}$ the category of left exact functors from $\mathbf{C}^{\prime}$ to $\mathbf{A b}$ ?

The following is an example where the answer to this question is positive.

Let $\mathbf{C}$ be a small category and let $J$ be a topology on $\mathbf{C}$ making ( $\mathbf{C}$, $J$ ) into a site (as in [5, Definition 1.2, pp. 256-303]). Let $\mathbf{A}$ be the category of sheaves of sets on $\mathbf{C}$, so that $\mathbf{A}$ is a full reflective subcategory of the functor category $(i, r): \mathrm{A} \rightarrow$ Sets $\mathrm{C}^{\circ \mathrm{P}}$, where $i: \mathrm{A} \rightarrow$ Sets $\mathrm{C}^{\circ \mathrm{p}}$ is the inclusion functor, then $\mathbf{R}$ is a left exact idempotent triple (where "left exact" means that the functor of $\mathbf{R}$ preserves finite limits).

Now Sets $C^{\circ p}$ is an example of an elementary topos (as in $[6, p .5]$ ) and one sees that the topologies $J$ on $\mathrm{C}$ are in one-to-one correspondence with the topologies on the elementary topos Sets ${ }^{\circ p}$ (as defined in [6]). One can then show (using [6, Proposition 3.22, p. 70]) that the assignment 
$J \mapsto \mathbf{R}$ as in the previous paragraph gives a one-to-one correspondence between topologies $J$ on $\mathbf{C}$ and left exact idempotent triples $\mathbf{R}$ on Sets ${ }^{\circ}$.

5. Theorem. Let $\mathbf{C}$ be a small category, J a topology on $\mathrm{C}, \mathrm{A}$ the category of sheaves of sets on $\mathbf{C}$ with respect to $J$, and $\mathbf{S}$ a cocontinuous triple on $\mathrm{A}$. Then there is a small category $\mathrm{C}^{\prime}$ and a topology $J^{\prime}$ on $\mathrm{C}^{\prime}$ so that $\mathbf{A}^{\mathbf{S}}$ is a category of sheaves of sets on $\mathbf{C}^{\prime}$ with respect to $J^{\prime}$.

Proof. Let $B=$ Sets ${ }^{\circ}{ }^{\circ}, R$ the left exact idempotent triple corresponding to $J, \mathbf{T}$ the triple on $\mathbf{B}$ induced by $\mathbf{S}$, and $\hat{\mathbf{T}}$ the cocontinuous approximation to $T$. Let $\mathbf{C}^{\prime}$ be a category for which $\mathbf{B}^{\hat{\mathbf{T}}} \cong \operatorname{Sets}^{\prime} \mathbf{C}^{\prime}$ (as in I). We have that $A^{\mathbf{S}} \cong\left(\mathbf{B}^{\hat{\mathbf{T}}}\right)^{\mathbf{R}}$, where $\widetilde{\mathbf{R}}$ is a lifting of $\mathbf{R}$. Now the underlying functor from $\mathbf{B}^{\hat{\mathbf{T}}}$ to $\mathbf{B}$ is not only faithful but preserves and creates limits. Therefore, since $\mathbf{R}$ is a left exact idempotent triple, $\widetilde{\mathbf{R}}$ must be also. We now let $J^{\prime}$ be the topology on $C^{\prime}$ corresponding to $\widetilde{\mathbf{R}}$, and we are done. $\square$

The referees of this paper have pointed out that Theorem 5 follows from Giraud's theorem (see [3, pp. 108-109]) in the following way. Since $\mathbf{S}$ is cocontinuous, the underlying functor $U: A^{S} \rightarrow A$ creates both limits and colimits. From this one sees that $A^{\mathbf{S}}$ is an exact category with limits, colimits, and disjoint universal sums. The free algebras in $A^{S}$ on the set of generators in $\mathbf{A}$ are easily seen to form a set of generators for $A^{S}$. Thus, by Giraud's theorem, $\mathbf{A}^{\mathbf{S}}$ is a topos, from which our Theorem 5 follows.

\section{REFERENCES}

1. M. Artin, Grothendieck topologies, mimeographed notes, Harvard University.

2. J. Beck, Distributive laws, Sem. on Triples and Categorical Homology Theory (ETH, Zürich, 1966/67), Springer, Berlin, 1969, pp. 119-140. MR 39 \#2842.

3. M. Barr, Exact categories, Lecture Notes in Math., vol. 236, Springer-Verlag, Berlin and New York, 1971, pp. 1-120.

4. J. Fisher-Palmquist and D. Newell, Triples on functor categories, J. Algebra 25 (1973), 226-258.

5. J. Giraud, Analysis situs, Séminaire Bourbaki, 1962/63, Fasc. 3, no. 256, Secrétariat mathématique, Paris, 1964, 11 pp. MR 33 \#1343.

6. A. Kock and G. C. Wraith, Elementary toposes, Lecture Notes Series, no. 30, Aarhus University, 1971.

7. S. Mac Lane, Categories for the working mathematician, Springer-Verlag, New York, 1971.

DEPARTMENT OF MATHEMATICS, UNIVERSITY OF CALIFORNIA, IRVINE, CALIFORNIA 92664 